\title{
Experimental demonstration of time-delay interferometry with optical frequency comb
}

\author{
Quentin Vinckier, ${ }^{1, *}$ Massimo Tinto®, ${ }^{1,2}$ Ivan Grudinin, ${ }^{1, \ddagger}$ Daniel Rieländer, ${ }^{1, \|}$ and Nan Yu $\odot^{1, \dagger}$ \\ ${ }^{1}$ Jet Propulsion Laboratory, California Institute of Technology, Pasadena, California 91109, USA \\ ${ }^{2}$ Also affiliated with Divisão de Astrofísica, Instituto Nacional de Pesquisas Espaciais, \\ S. J. Campos, SP 12227-010, Brazil \\ and University of California San Diego, Center for Astrophysics and Space Sciences, \\ La Jolla, California 92093, USA
}

(Received 14 April 2020; accepted 31 July 2020; published 9 September 2020)

\begin{abstract}
Heterodyne laser phase measurements in the Laser Interferometer Space Antenna (LISA) are degraded by the phase fluctuations of the onboard clocks, resulting in unacceptable sensitivity performance levels of the interferometric data. The current scheme for cancellation of the clock phase noise requires $1 \mathrm{GHz}$ modulation of the ranging laser beams and additional interspacecraft clock recovery heterodyne phase measurements. Here, we report experimental results for an alternative approach to clock noise cancellation based on modified second generation time-delay interferometry (TDI) with optical frequency combs (OFCs). The use of OFCs in the LISA scheme allows simultaneous cancellation of both laser and clock noises, and would eliminate the need for $1 \mathrm{GHz}$ laser modulations and associated demodulation detections. Two Mach-Zehnder interferometers with acousto-optic modulators were used to simulate two LISA arms with Doppler shifts and time delays. With a self-referenced OFC locked to the laser providing the clock signal, we achieve simultaneous suppression of laser and clock noises by 7 and 1.5 orders of magnitudes, respectively, down to the setup noise floor. Based on a numerical analysis, we further show that the noise suppression performance of the OFC-based TDI scheme can meet the LISA mission requirements with an ample margin.
\end{abstract}

DOI: 10.1103/PhysRevD.102.062002

\section{INTRODUCTION}

Gravitational waves (GWs), predicted by Einstein's theory of general relativity [1,2], are space-time oscillations propagating at the speed of light. They are induced by highly energetic cosmic events, such as coalescences and mergers of binary black holes and neutron stars, supernovae explosions, and gamma-ray bursts. Their first detection in September 2015 by the two detectors of the Laser Interferometer Gravitational-wave Observatory (LIGO) marks the beginning of gravitational wave astronomy, opening a new window on the Universe for surveying cosmic events otherwise impossible to observe with conventional telescopes [3,4]. LIGO was designed to cover a spectrum ranging from a few tens of $\mathrm{Hz}$ to a few $\mathrm{kHz}$. The lower bound of this spectral range is limited by the Earth's seismic and gravity-gradient noises.

\footnotetext{
*vinckier@jpl.nasa.gov

†Nan.Yu@jpl.nasa.gov

${ }^{\star}$ Now affiliated with Beamlet, South Pasadena, California 91031, USA

"Now affiliated with Fraunhofer Institute for Applied Optics and Precision Engineering IOF, 1207745 Jena, Thuringia, Germany
}

In order to access lower regions of the GW spectrum, the European Space Agency (ESA) and the National Aeronautics and Space Administration (NASA) are planning to jointly fly the Laser Interferometer Space Antenna (LISA), expected to be ready for launch around 2030 [5]. LISA is a space-based interferometer consisting of three interplanetary spacecraft flying in an almost equilateral triangle configuration with exchanging coherent laser beams along its three arms of 2.5 million $\mathrm{km}$. It is designed to cover a bandwidth from $10^{-4}$ to $10^{-1} \mathrm{~Hz}$, unveiling a broad variety of GW sources unobservable by groundbased detectors [6]. The phase fluctuations induced by a GW are measured by interfering the incoming laser beam with the outgoing laser beam on each spacecraft. Because the length of each arm is much larger than the coherence length of the stabilized LISA lasers, the laser noise dominates over the GW signal in heterodyne one-way measurements by 6 to 11 orders of magnitude [6-8]. Although LISA employs two nearly equal arms, the arm length difference can only be maintained with a precision of about a percent from the nominal length [9]. This is not enough for the laser noise to cancel to the required level. To address this problem, it has been shown that, through knowledge of the length of each LISA arms, it is 
possible to delay and linearly combine the phase measurements from each arm to cancel the laser noise to a sufficiently low level and observe gravitational radiation signals. This postprocessing technique is called time-delay interferometry (TDI), and any possible linear combination is called a TDI observable [10].

On the other hand, because of the relative interspacecraft velocities, the received laser beams are Doppler shifted and their interferences with the onboard laser produces beat notes of up to $25 \mathrm{MHz}$ [5]. To measure these heterodyne signals and recover the GW signal in the TDI measurements, the LISA baseline design relies on onboard, ovenstabilized crystal clocks, usually referred to as ultrastable oscillators (USOs). The space-qualified USO baselined by the LISA project is characterized by a phase noise level estimated to be up to 5 orders of magnitude higher than the LISA noise requirements at $10^{-4} \mathrm{~Hz}$, when considering $20 \mathrm{MHz}$ beat notes $[6,8,11,12]$. To suppress the USO noises, the current LISA measurement scheme requires the transfer of the USO noises between the spacecraft by frequency modulating each laser beam exchanged by the spacecraft [11-14]. By using six additional phase measurements obtained by comparing the sidebands of the incoming against the outgoing laser beams, specific combinations of the carrier-to-carrier and sideband-to-sideband measurements allow one to calibrate out the clock noise from the TDI observables [11-17].

In 2015, Tinto and Yu proposed a new TDI scheme to simultaneously cancel the laser and clock noise by using optical frequency combs (OFCs) [18]. Leveraging the recent advent of self-referenced OFC as an optical frequency divider [19-21], they suggested to lock an OFC to the onboard laser and use the OFC repetition rate signal at radio frequency (rf) as the clock signal for the heterodyne phase measurements. They theoretically showed that, because the local oscillator is phase coherent with the onboard laser, a modified second-generation TDI combination can be used to suppress both laser and clock noises. This approach leads to a significant simplification of the onboard interferometer measurement subsystem. Indeed, the $1 \mathrm{GHz}$ interferometric laser beam modulation and additional heterodyne measurements involving microwave sidebands would no longer be needed. The entire onboard USO system would no longer be needed and be replaced by the OFC referenced to the onboard laser. This alternative interferometer scheme would significantly simplify the implementation of TDI with the heterodyne measurements, thus significantly reduce the probability of subsystem failures.

In this paper, we describe an experimental proofof-concept demonstration of the optical frequency combbased TDI. With a tabletop experiment setup of two 2-way laser interferometers with simulated Doppler frequency shifts and time delays, and a self-referenced OFC, we achieve 7 orders of magnitude laser noise suppression without the need of any independent clock signal over the
$10^{-4}$ to $10^{-1} \mathrm{~Hz}$ LISA frequency bandwidth, limited only by the experiment measurement noise floor. We further show through numerical simulation that using a selfreferenced OFC phase locked to the onboard ranging laser in the two-arm two-way interferometer scheme, both laser and heterodyne local oscillator rf noises can be suppressed well below the LISA sensitivity requirements.

This article is organized as follows. In Sec. II, we present the basics of how an OFC works together with a summary of the implementation of TDI with OFC. The experimental setup is then described in Sec. III, while the corresponding results are discussed in Sec. IV. In Sec. IV, based on a numerical analysis, we also quantify the expected performance of TDI with OFC when implemented in the actual LISA scenario. In Sec. V, we present our conclusions and emphasize that the use of the OFC technique in the context of LISA would result in hardware simplification and improved mission reliability.

\section{PRINCIPLE OF OFC-BASED TDI}

\section{A. Self-referenced optical frequency combs}

An optical frequency comb is an optical oscillator that generates a coherent pulse train in the time domain at a stable frequency $f_{\text {rep. }}$. In the frequency domain, it corresponds to a series of $n$ equally spaced narrow frequency lines of frequency $\nu_{n}$, each of these modes being phase coherent. The spacing between each mode is equal to $f_{\text {rep }}$. Because of dispersion, each mode does not have exactly the same phase velocity, inducing a phase offset between the optical carrier and the pulse envelope that changes over time. This offset is called carrier envelope offset (CEO) phase, and the rate of this offset phase change from pulse to pulse is called the CEO frequency $\nu_{\mathrm{CEO}}$. The description of an OFC in the spectral domain is given by the following simple and well-known relation [22]:

$$
\nu_{n}=\nu_{\mathrm{CEO}}+n f_{\text {rep }} .
$$

In practice, the two frequencies $\nu_{\mathrm{CEO}}$ and $f_{\text {rep }}$ can be controlled through proportional integral derivative (PID) regulation feedback loops. When the OFC has an octave spanning width, it is possible to measure directly $\nu_{\mathrm{CEO}}$ by frequency doubling one low frequency mode $\nu_{n^{\prime}}$ and beating this signal with the OFC mode of frequency $2 \nu_{n^{\prime}}$. Then the measurement of $\nu_{\mathrm{CEO}}$ can be used to stabilize $\nu_{\text {CEO }}$ itself, resulting in a "self-referenced OFC [23]."

Equation (1) shows that when $\nu_{\mathrm{CEO}}$ is self-referenced, there is a coherent phase relationship between the optical signals of frequency $\nu_{n}$ and the microwave signal of frequency $f_{\text {rep. }}$. Thus, if the OFC is locked to an external laser by adjusting $f_{\text {rep }}$ so as to maintain a stable beat note between the laser signal and one comb tooth, the OFC then becomes an optical frequency divider. It coherently transfers, with extreme precision, the laser phase 
fluctuations down to the microwave band. The level of precision of this down-conversion process has been demonstrated experimentally to be smaller than the frequency white noise associated with the experimental setup used to measure it [24] and estimated to correspond to an Allan standard deviation of about $\sigma_{\tau}=10^{-19}$ at an integration time $\tau=1000 \mathrm{sec}$. Since the corresponding power spectrum of the relative frequency fluctuation is given in terms of the Allan standard deviation by the following expression [25]:

$$
S_{\nu}(f)=2 \tau \sigma_{\tau}^{2} \approx 10^{-35} \mathrm{~Hz}^{-1},
$$

we conclude this noise spectrum to be 9 to 13 orders of magnitude smaller than the spectrum of a typical USO noise over the frequency band of interest to space-based GW detectors [12]. In the context of the experiment presented in this paper, we exploit this phenomena by locking our OFC to the outgoing ranging laser and using the $\mathrm{OFC}$ repetition rate signal as reference clock for the heterodyne beat notes measurements. The correlation between the laser phase noise and that of the clock allows us to use a new TDI combination to suppress both laser and clock noises in one step, as will be shown below.

While self-referenced OFCs are now commercially available, recent advancements have also resulted in space-borne OFCs [19-21]. Moreover, significant progress has also been made on miniaturized OFC based on nonlinear microresonators $[26,27]$. It is therefore reasonable to expect space-qualified, power-efficient OFCs of small form factors to become available in the near future.

\section{B. Time-delay interferometry with self-referenced optical frequency combs}

LISA is a triangular, nearly equal-arm, interferometer that relies on three spacecraft in heliocentric orbits, each carrying two lasers [5]. The three spacecraft are separated by a nominal distance of 2.5 million $\mathrm{km}$ and the arm length variations are maintained below $1 \%$ through monthly station keeping maneuvers [9]. Because the lasers are coherently coupled $[8,28]$, we may regard two arms of the LISA laser interferometer configuration as being equivalent to that of two 2-way arms with a single laser onboard one spacecraft that emits light towards two separated mirrors moving at different velocities, as illustrated in Fig. 1. The beat notes at the detectors $y_{i}(t)$ are given by

$$
\begin{aligned}
y_{i}(t)= & \nu_{0}\left(1-\frac{2 V_{i}}{c}\right)-\nu_{0}+\nu_{\text {noise }}\left(t-T_{i}\right)\left(1-\frac{2 V_{i}}{c}\right) \\
& -\nu_{\text {noise }}(t)+h_{i}(t),
\end{aligned}
$$

where $\nu_{0}$ is the ranging laser center frequency, $\nu_{\text {noise }}(t)$ its frequency noise, $V_{i}$ the Doppler velocity, $T_{i}$ the time delay

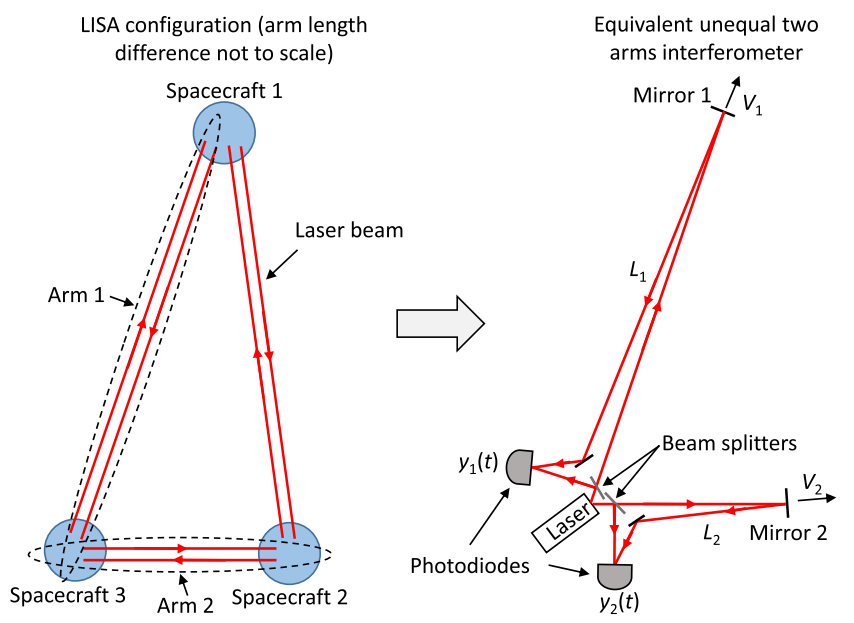

FIG. 1. Simplified unequal-arm Michelson interferometer (right) from the three-arm LISA triangular configuration (left). Each mirror moves at a velocity $V_{i}$ relative to the inertial reference frame with respect to which the laser and the beam splitter are at rest, and the arm-length is $L_{i}$. In the case of the LISA array, $V_{i}$ and $L_{i}$ are slowly varying functions of time and can be regarded as constants over a period of a day or so. For each arm, the laser beam back reflected by the mirror is combined with the outgoing laser beam at a photodiode where phase or frequency fluctuations $y_{i}(t)$ are measured.

corresponding to twice the arm length $L_{i}, c$ the speed of light, and $h_{i}(t)$ the gravitational signal. ${ }^{1}$

With the $1 \%$ arm length difference, however, it still requires a frequency stability of the laser that is way beyond that of any state of the art stabilized laser system. As a consequence, the TDI scheme is used to suppress the laser frequency noises. The conventional TDI scheme has been discussed and tested extensively in literature [7,10-13, 15-17,28]; here, we will only describe how the OFC is applied to accomplish the second generation TDI, and discuss how it is applied in the experimental demonstration in this paper. We refer readers to Ref. [13] for details of experimental hardware implementation comparisons.

The laser beat notes $y_{i}(t)$ are measured with the heterodyne detection scheme as shown in Fig. 2. Here, the reference clock is provided by a self-referenced OFC rather than an independent USO. All relevant symbols and designations are clearly described in the figure caption. The frequency counter operates in a closed feedback loop such that the output of the mixer is nulled to the detection noise limit (which is not considered at this moment). When the loop is closed properly, we have

$$
y_{i}(t)-a_{i}(t) f(t)=0,
$$

\footnotetext{
${ }^{1}$ The $y_{i}(t)$ retains the main Doppler beat tone $\Delta \nu_{i}=\left(\frac{2 V_{i}}{c}\right) \nu_{0}$ for the experimental data analysis reasons in this work while it is normally removed from the term of $y_{i}(t)$ in LISA TDI analysis in the literature.
} 


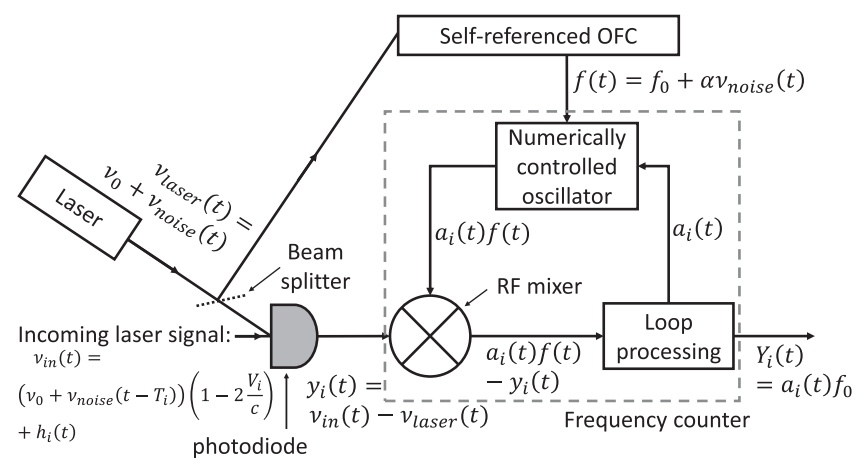

FIG. 2. Block diagram of the signal flow for one arm of the unequal-arm interferometer heterodyne measurement with the OFC implementation. $\nu_{\text {laser }}(t)$ is the laser frequency with $\nu_{0}$ the center frequency and $\nu_{\text {noise }}(t)$ the frequency noise, $\nu_{\text {in }}(t)$ the frequency of the back reflected laser signal, $T_{i}$ the time delay corresponding to twice the arm length $L_{i}, c$ the speed of light, $\frac{2 V_{i}}{c}$ the Doppler frequency shift factor of the back reflected laser signal, $h_{i}(t)$ the gravitational wave signal, $f_{0}$ the clock reference frequency derived from the optical frequency $\nu_{0}$ through the OFC and additional mixer (see Sec. III for more details). $f(t)=$ $f_{0}+\alpha \nu_{\text {noise }}(t)$ is the clock frequency provided to the frequency counter, $\alpha=\frac{\partial f(t)}{\partial \nu_{\text {laser }}(t)}$ the optical frequency division factor of the OFC between the laser optical signal and the microwave repetition rate signal of the OFC, and $a_{i}(t)$ the gain coefficient of the feedback loop adjusted such that $y_{i}(t)-a_{i}(t) f(t)=0$. The output of the loop processing is the measurement of $y_{i}(t)$, designated as $Y_{i}(t)$.

where $f(t)$ is the clock frequency to the counter derived from the repetition rate signal of the OFC and $a_{i}(t)$ the gain coefficient of the feedback loop. $f(t)$ has the center frequency $f_{0}$ with the noise $f_{\text {noise }}(t)$ that is coherently transferred from the optical noise $\nu_{\text {noise }}(t)$. The relationship between $f_{\text {noise }}(t)$ and $\nu_{\text {noise }}(t)$ are proportional and can be expressed as $f_{\text {noise }}(t)=\alpha \nu_{\text {noise }}(t)$ as discussed in the previous section, and thus,

$$
f(t)=f_{0}+\alpha \nu_{\text {noise }}(t),
$$

where $\alpha=\frac{\partial f(t)}{\partial \nu_{\text {laser }}(t)}$ is the optical frequency division factor of the OFC. ${ }^{2}$ We will designate the actual counter read-out (output) for measured $y_{i}(t)$ as $Y_{i}(t)$, which is then given by

$$
Y_{i}(t)=a_{i}(t) f_{0} .
$$

By injecting $f_{0}=f(t)-\alpha \nu_{\text {noise }}(t)$ [see Eq. (5)] in Eq. (6), we have

$$
Y_{i}(t)=y_{i}(t)-a_{i}(t) \alpha \nu_{\text {noise }}(t) .
$$

\footnotetext{
${ }^{2}$ In Tinto and $\mathrm{Yu}, \alpha=\frac{\partial f(t)}{\partial \nu_{\text {aser }}(t)}=\frac{f_{0}}{\nu_{0}}[18]$. However, as we will see in the next sections, it is not the case for our experiment because of the commercial OFC system used.
}

Recall that $a_{i}(t)=Y_{i}(t) / f_{0}$ [see Eq. (6)], we obtain

$$
Y_{i}(t)=\frac{1}{1+\alpha \frac{\nu_{\text {noise }}(t)}{f_{0}}} y_{i}(t) .
$$

Considering $f_{0} \gg \alpha \nu_{\text {noise }}(t)$ (in our case $|\alpha| \sim 10^{-6}$, $f_{0}=10^{7} \mathrm{~Hz}$, and the noise of the stabilized LISA laser is of the order of $\left.\sim 300 \frac{\mathrm{HZ}}{\sqrt{(\mathrm{Hz})}}[8]\right)$, to first order we have

$$
Y_{i}(t)=\left[1-\alpha \frac{\nu_{\text {noise }}(t)}{f_{0}}\right] y_{i}(t) \text {. }
$$

By substituting Eq. (3) in Eq. (9) and approximating $\nu_{\text {noise }}\left(t-T_{i}\right)\left(1-\frac{2 V_{i}}{c}\right) \simeq \nu_{\text {noise }}\left(t-T_{i}\right)$, we have

$$
\begin{aligned}
Y_{i}(t)= & {\left[1-\alpha \frac{\nu_{\text {noise }}(t)}{f_{0}}\right]\left[\Delta \nu_{i}+\nu_{\text {noise }}\left(t-T_{i}\right)\right.} \\
& \left.-\nu_{\text {noise }}(t)+h_{i}(t)\right] .
\end{aligned}
$$

Typically, $\Delta \nu_{i} \in[5,25] \mathrm{MHz}[5,8]$. By neglecting noise quadratic terms and the product between the laser noise and the GW signal $h_{i}(t)$, Eq. (10) becomes equal to

$Y_{i}(t)=\Delta \nu_{i}+\nu_{\text {noise }}\left(t-T_{i}\right)-\nu_{\text {noise }}(t)+h_{i}(t)+q_{i}(t)$,

$$
q_{i}(t) \simeq-\alpha\left(\frac{\Delta \nu_{i}}{f_{0}}\right) \nu_{\text {noise }}(t) \simeq-\alpha a_{i}(t) \nu_{\text {noise }}(t),
$$

where $q_{i}(t)$ is the clock noise in the heterodyne measurements clearly down-converted from the laser noise. As also discussed in Tinto and $\mathrm{Yu}$ [18], Eq. (12) shows that $q_{i}(t)$ and $\nu_{\text {noise }}(t)$ are proportional, which makes the simultaneous noise cancellation possible through combination. Based on Eqs. (11) and (12), it is then easy to show that the following second generation TDI combination preserves the gravitational wave signal $h_{i}(t)$ while canceling both the clock noise $q_{i}(t)$ and the laser noise $\nu_{\text {noise }}(t)$ :

$$
\begin{aligned}
X^{\mathrm{OFC}}(t) \equiv & {\left[Y_{1}\left(t-T_{2}\right)-\left(1+\alpha \frac{\Delta \nu_{2}}{f_{0}}\right) Y_{1}(t)\right] } \\
& -\left[Y_{2}\left(t-T_{1}\right)-\left(1+\alpha \frac{\Delta \nu_{1}}{f_{0}}\right) Y_{2}(t)\right] .
\end{aligned}
$$

\section{EXPERIMENTAL IMPLEMENTATION}

There are two key aspects of the LISA laser interferometer measurements that must be properly implemented in the experimental setup to demonstrate the suppression of both laser and local oscillator noises. The first is the Doppler frequency shift of the returned laser beams, which 
is simulated in our case by acousto-optic modulators $(\mathrm{AOM})$. The second is the time delay corresponding to the long LISA interferometer arms that is of the order of light seconds. Such a long delay is impossible to implement in a laboratory. To properly simulate an arbitrary laser propagation time delay, we came up with the approach of applying a known noise sequence to the outgoing laser beam and the same noise sequence delayed by a time $T_{i}$ to the returned laser beams. Since AOMs are used to simulate the Doppler frequency shifts, the applied laser noise can be added through the corresponding AOM. Our setup is depicted in Fig. 3. It is an experimental implementation of the simplified unequal-arm Michelson interferometer presented in Fig. 1. In Fig. 3, the onboard outgoing laser beam is marked in green, and the returned laser beams are marked in blue. Each laser beam passes through an AOM through which both Doppler frequency shift and laser frequency noise $\nu_{\text {noise }}(t)$ and $\nu_{\text {noise }}\left(t-T_{i}\right)$ are applied. The total Doppler shift $\Delta \nu_{i}$ of each returning laser beam will be the differences of the AOM operation frequencies in the outgoing laser beam and the corresponding returned beam. Three identical synthesizers are used to drive the AOMs. These synthesizers are controlled and programmed by a computer to generate the frequency noise sequences of the desired noise spectrum and delays. All synthesizers are referenced to a common oscillator, a hydrogen maser. The low phase noise performance of the maser (fractional frequency instability is less than $10^{-13} \times \tau^{-1 / 2}$ from $\tau=1 \mathrm{~s}$ to $\tau=10^{4} \mathrm{~s}$ ) is necessary and guarantees negligible phase noise impact from the oscillator in the time delayed analyses.

The interference of the outgoing laser beam with each of the returned laser beams is detected by a photodetector. Since the Mach-Zehnder interferometers in the setup shown in Fig. 3 are near equal-arm interferometers with little path length difference, the intrinsic laser noise appears as a correlated common noise source on both the outgoing and returned laser beams and cancels at the photodetector. Thus the residual frequency noises at the detectors are only limited by the stability of the interferometers themselves. The interferometers are enclosed in a box to minimize environmental disturbances (airflow through the arms), and the setup is mounted on a pneumatic antivibration optical table. The output signal of each photodetector $y_{i}(t)$ is measured using a commercial two-channel frequency counter that yields the frequency counter measurement $Y_{i}(t)$ as depicted in Fig. 2.

A part of the outgoing laser beam marked in green in Fig. 3 is combined with the OFC optical output that is filtered around $\nu_{0}=193.4 \mathrm{GHz}(1550 \mathrm{~nm})$. The beat note

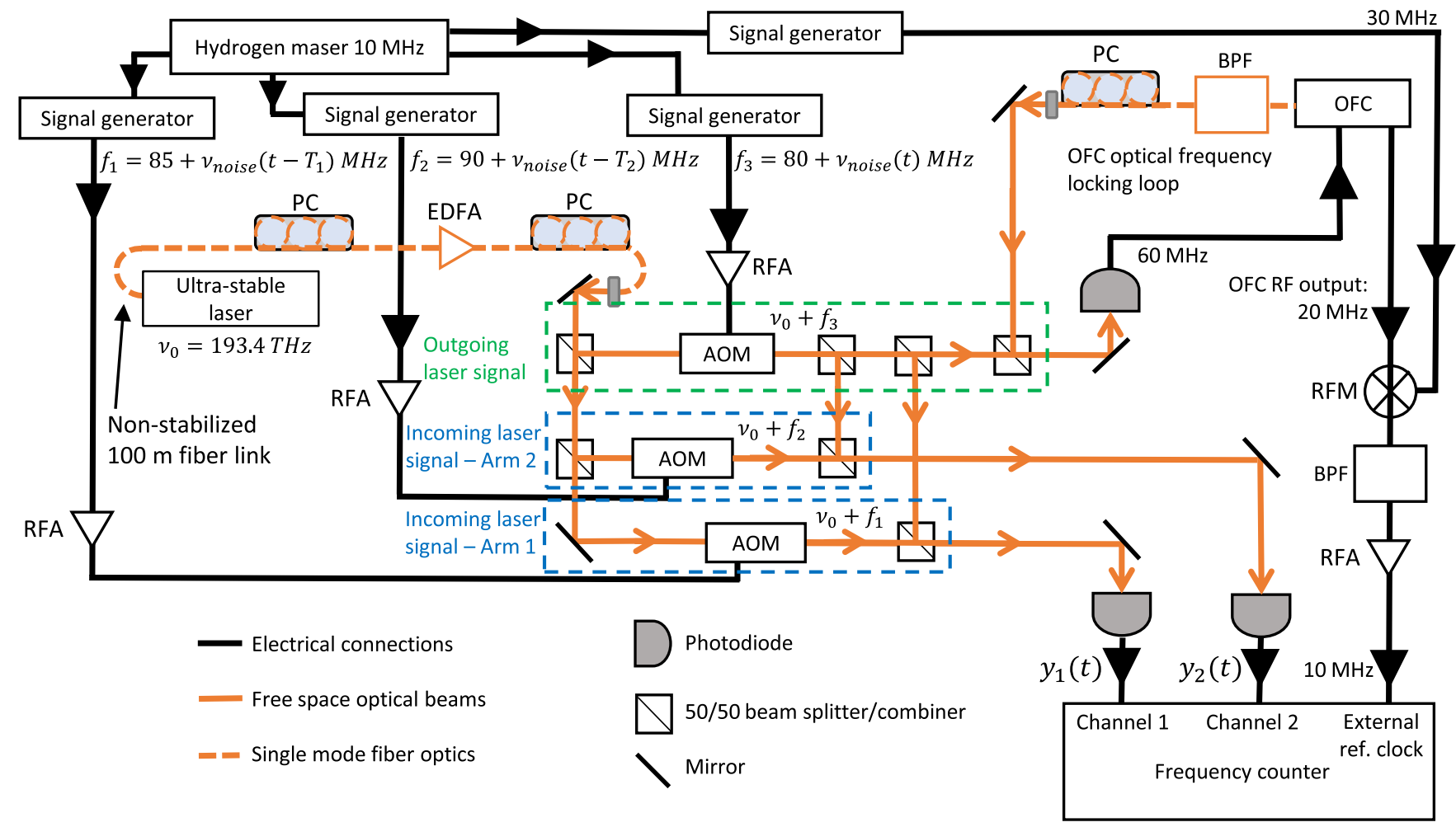

FIG. 3. Experimental setup. OFC: Optical frequency comb, BPF: Band pass filter, RFA: Radio frequency amplifier, RFM: Radio frequency mixer, AOM: Acousto-optic modulator, PC: Polarization controller, and EDFA: Erbium-doped fiber amplifier. The ultrastable laser signal is brought into the experimental setup from another lab through a $100 \mathrm{~m}$ fiber link. The two 2-way laser interferometer arms in Fig. 1 are experimentally represented in the dashed line boxes: the green box simulates the onboard outgoing laser beams while the blue boxes the returning incoming laser beams. 
produced by this interference is stabilized at an offset frequency of $60 \mathrm{MHz}$ using a proportional integral derivative (PID) controller loop that adjusts the OFC repetition frequency $f_{\text {rep }}(t)$. Ideally, as discussed in Tinto and Yu [18], the OFC should work as a pure frequency divider that directly provides the required $\mathrm{rf}$ frequency value $f_{0} \in$ $[9.75,10.25] \mathrm{MHz}$ from the optical frequency $\nu_{\text {laser }}(t)$ with a simple division ratio $([9.75,10.25] \mathrm{MHz}$ is the range of the reference frequency input of the counter we use). However, the commercial OFC system that we use is a general purpose system with an internal frequency synthesis chain. The repetition rate of the OFC system is approximately equal to $250 \mathrm{MHz}$. The actual rf output of the comb is at $20 \mathrm{MHz}$, internally synthesized by mixing the fourth harmonics of the repetition rate with an external referenced $980 \mathrm{MHz}$ signal. Since the frequency counter only accepts about $10 \mathrm{MHz}$, the comb $20 \mathrm{MHz}$ is further mixed with a $30 \mathrm{MHz}$ signal source. The final input of the reference frequency to the counter is, therefore,

$$
f(t)=30-\left[4 f_{\text {rep }}(t)-980\right] \mathrm{MHz} .
$$

As previously mentioned, the $\mathrm{OFC}$ is locked to the laser by maintaining a $60 \mathrm{MHz}$ beat note between one OFC frequency mode and the laser signal,

$$
\nu_{\mathrm{CEO}}+n f_{\text {rep }}(t)-\nu_{\text {laser }}(t)=60 \mathrm{MHz} .
$$

Based on Eqs. (14) and (15), it follows that $\alpha=\frac{\partial f(t)}{\partial \nu_{\text {laser }}(t)}=$ $-\frac{4 f_{\text {rep }}(t)}{\nu_{0}}=-\frac{1 \mathrm{GHz}}{193399.7 \mathrm{GHz}}=-5.171 \times 10^{-6}$.

Note that the rf sources mentioned in the frequency synthesis, that is, $60 \mathrm{MHz}, 30 \mathrm{MHz}$, and $980 \mathrm{MHz}$, are all phase locked to the hydrogen maser, and therefore, their phase noise contributions to the final measurement results are negligibly small. In the LISA application, a proper customized design for the OFC and the frequency counter would no longer require the rf frequency mixing and synthesis, and the use of the hydrogen maser described above in this experiment. It should also be pointed out that the intrinsic laser frequency noise itself also gets transferred to the OFC repetition rate and hence, the clock signal to the counter. This noise is not correlated with the time delays as those from the simulated laser noise and therefore, will not be canceled in the TDI combination. It must be reduced to below experiment measurement noise floor. For this reason, an ultrastable laser is used to minimize the laser intrinsic frequency noise in the demonstration experiment setup. This of course will not be the case and presents no limitation in the actual LISA scenario with the real noise delays.

The synthesizers driving the AOMs and the frequency counter are controlled via a Python code. The Python code outputs the measurements $Y_{i}(t)$ in a file that is read and postprocessed with MATLAB to perform the TDI combination and analyze the results. The sampling time of the frequency counter is set at $\sim 2.7 \mathrm{sec}$., and the following experimental parameters were implemented: $T_{1}=2.7 \mathrm{sec}$., $T_{2}=5.4 \mathrm{sec} ., \Delta \nu_{1}=5 \mathrm{MHz}$, and $\Delta \nu_{2}=$ $10 \mathrm{MHz}$. Note that the delays $T_{i}$ used do not correspond to those of the actual LISA mission $(\approx 17 \mathrm{sec})$, but it does not influence the TDI validation and results. Indeed, Eq. (13) demonstrated in Sec. II is valid for any delay values $T_{1}$ and $T_{2}$.

\section{RESULTS}

\section{A. Experimental measurement noise floor}

We first evaluate the experiment setup measurement noise floors. To perform these measurements, all rf sources are phase locked to the hydrogen maser, including the $10 \mathrm{MHz}$ reference to the frequency counter. That is, the OFC is not used. Furthermore, no simulated noise is applied to the AOMs. In this condition, the measurements $Y_{i}(t)$ give the frequency noise floors of the two interferometers formed by the "outgoing" laser arm and the "returning" laser arms. The beat notes time sequences are converted into the spectral domain via a fast-Fourier transform algorithm as plotted in Fig. 4.

The frequency noise measurements are below $10^{-4} \frac{\mathrm{Hz}}{\sqrt{(\mathrm{Hz})}}$ in the frequency range of the interest and plateau at $1 \mathrm{mHz}$ and below. It is a combination of the instability of the interferometers and the noise of the commercial frequency counter used. We can also use these data to determine the noise floor of the postprocessed second generation TDI by using Eq. (13) for $\alpha=0$, $T_{1}=2.7 \mathrm{sec}$., and $T_{2}=5.4 \mathrm{sec}$. The result is also given in Fig. 4. The TDI processed noise is always below that

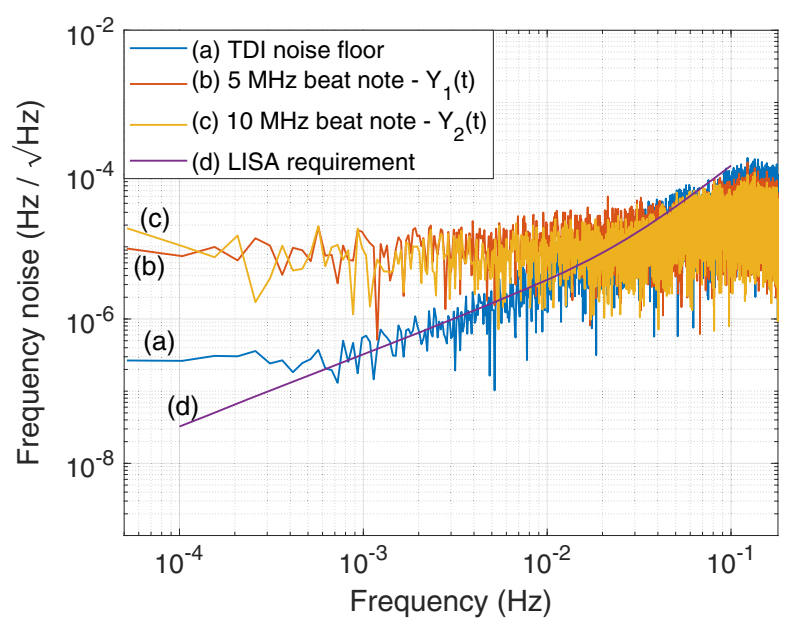

FIG. 4. Experimental setup noise floor measurements. Both red (b) and yellow (c) curves are the noise spectral amplitudes for $Y_{1}(t)$ and $Y_{2}(t)$, respectively. The TDI postprocessed noise floor is plotted in blue (a). The frequency noise LISA requirement shown in purple (d) is derived from [6]. 
of the direct heterodyne measurements $Y_{i}(t)$. This is explained by the delay line transfer function [29,30]. The frequency noise requirement for LISA is also plotted in Fig. 4 from Ref. [6].

\section{B. Verification of the frequency division coefficient $\boldsymbol{\alpha}$}

To ensure the OFC coherently transfers the laser frequency noise to the rf domain, we experimentally verified the coupling between the laser and the clock signal. In this measurement, the OFC is phase locked to the laser beam of the outgoing arm. The counter reference clock signal is derived from the OFC as in Fig. 3 and described before. A sequence (7111 values of a Gaussian white noise with a standard deviation of $50 \mathrm{kHz}$ ) of known applied frequency shift steps $\left[\nu_{\text {noise }}(t)\right]$ is applied to all AOMs without any time delay $\left(T_{i}=0\right)$ so that the beat notes $y_{1}(t)$ and $y_{2}(t)$ are unaffected by the optical frequency changes. On the other hand, the OFC transfers the resulting optical frequency changes per applied frequency steps to the rf reference clock frequency to the counter. As such, the counter output $\left[Y_{1}(t)\right]$ yields the changes in the rf clock frequency. The resulting plot of the rf frequency change as a function of the applied optical frequency change is presented in Fig. 5.

A linear line fit yields the slope $\alpha=-5.173 \times 10^{-6}$. The minus sign is due to the frequency down mixing of the OFC $20 \mathrm{MHz}$ microwave signal with a $30 \mathrm{MHz}$ signal (see Sec. III). This is very consistent with the expected value of $\alpha=-5.171 \times 10^{-6}$. A close examination shows that there is a small variance between the measured frequency values and the expected ones, which is largely due to the frequency shifts of the ultrastable laser we used.

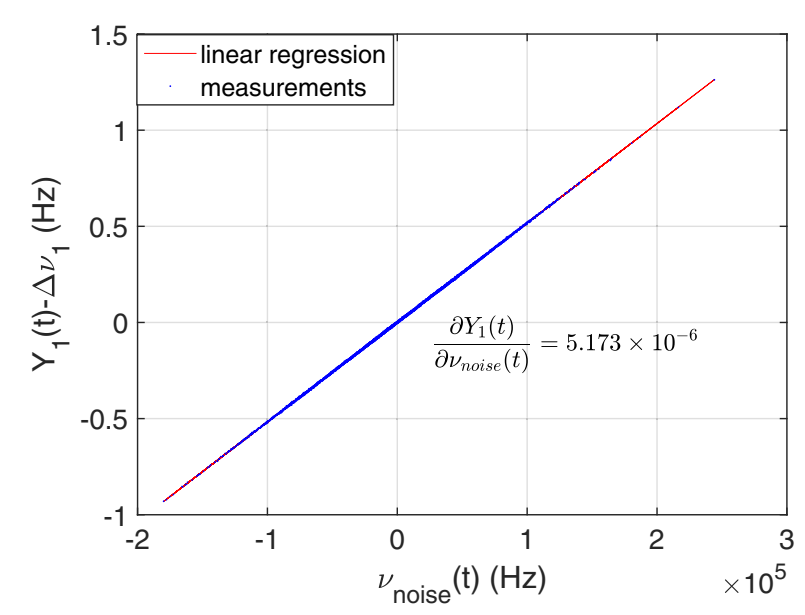

FIG. 5. Experimental evaluation of the coupling coefficient $\alpha$. The horizontal axis is the applied frequency steps in optical frequency domain while the vertical axis shows the resulting beat note frequency changes due to the counter rf reference frequency changes through the OFC.

\section{Conventional TDI performance evaluation}

Before demonstrating the comb-based TDI, we establish the baseline performance with the conventional TDI in the experiment setup. This can be done by removing the OFC system from the scheme and use the hydrogen maser as the counter clock input. The phase noise of the maser is extremely low, and the clock noise will not show up above the measurement noise floor. This is justified since in the current LISA scheme, the TDI for suppressing clock noise is independent of that of the laser noise. We apply simulated laser noise with a Gaussian noise statistics with the rms noise amplitudes of $50 \mathrm{kHz} . Y_{i}(t)$ are measured with $T_{1}=2.7 \mathrm{sec}$., $T_{2}=5.4 \mathrm{sec}$., and $\alpha=0$. The results of the measurements and the TDI postprocessed data are plotted in Fig. 6. As expected, we were able to completely suppress the laser noise to the experiment measurement noise floor. The simulated noise spectra and those of the direct measurement $Y_{i}(t)$ are also given in Fig. 6 for reference. This shows that the TDI demonstration setup is capable of realizing the TDI laser noise suppression by over 7 orders of magnitude.

On the other hand, if we use a noisier quartz oscillator for the clock reference input, we would expect that the clock noise will not be canceled and shows up in the measurements. To illustrate this point, we have performed the same measurements as above but using a clock signal provided by an independent $10 \mathrm{MHz}$ quartz oscillator instead of the hydrogen maser. The results are presented in Fig. 7 together with the measured noise spectrum of the oscillator itself.

Figure 7 clearly shows that TDI postprocessing performance is limited by the clock noise: the noise level of the TDI postprocessed signal at $10^{-1} \mathrm{~Hz}$ matches the one of the clock, which means that the clock noise is not canceled. Moreover, the noise floor of the TDI postprocessed signal

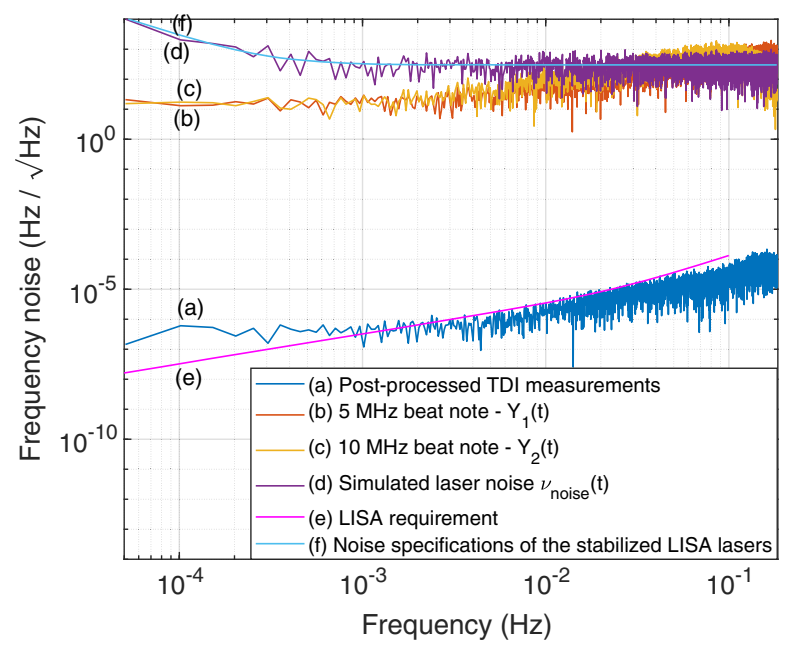

FIG. 6. TDI experimental results with the clock signal provided by the hydrogen maser. The LISA requirement is published in [6], and the noise spectrum of the stabilized LISA laser is provided in [8]. 


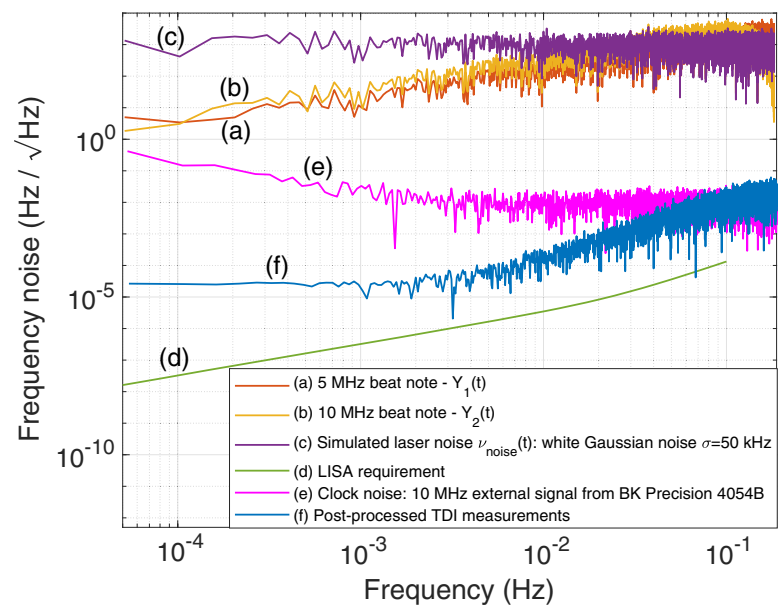

FIG. 7. TDI experimental results with the clock signal provided by an external noisy signal uncoupled from the laser.

in Fig. 7 is above $10^{-5} \frac{\mathrm{Hz}}{\sqrt{(\mathrm{Hz})}}$ at frequencies $<10^{-3} \mathrm{~Hz}$, whereas it would be below $10^{-6} \frac{\mathrm{Hz}}{\sqrt{(\mathrm{Hz})}}$ if the clock noise was canceled (see Figs. 4 and 6).

\section{OFC-based TDI demonstration}

To demonstrate the simultaneous laser and clock noise suppression with the use of OFC, we proceed with the same measurements but use the OFC repetition rate signal and $\mathrm{rf}$ synthesis to generate the clock signal for the frequency counter as presented in Fig. 3. However, in order to measure the clock noise suppression, the clock signal was directly plugged into one of the input channels of the frequency counter, with the clock signal provided by the hydrogen maser, and the same time series was used to generate $\nu_{\text {noise }}(t)$. Figure 8 plots the measured comb-based clock noise, together with the applied noise spectrum of $\nu_{\text {noise }}(t)$. It clearly shows the coherent coupling between the clock signal and the laser signal, with a factor of $|\alpha|=5.17 \times 10^{-6}$ between the two signals.

The OFC-based TDI results presented in Fig. 8 shows a laser noise cancellation factor of 7 orders of magnitude between the TDI postprocessed measurements and the measured beat notes $Y_{i}(t)$, together with the suppression of the clock noise, recovering the TDI measurement noise floor of our experiment setup reported in Fig. 4.

The TDI results of noise cancellation demonstrated meet the LISA requirements for frequencies $>3 \times 10^{-3} \mathrm{~Hz}$. The noise floor of our test bed is a combination of two artifacts that are not present in the actual implementation and operation of LISA: interferometer instability due to (1) airflow and (2) thermally unstabilized setup, and free-running laser noise uncoupled in the beat notes but coupled in the clock signal. In order to experimentally demonstrate further the LISA noise requirement on the entire $\left[10^{-4}, 10^{-1}\right] \mathrm{Hz}$ LISA bandwidth, the Mach-Zehnder interferometer noise

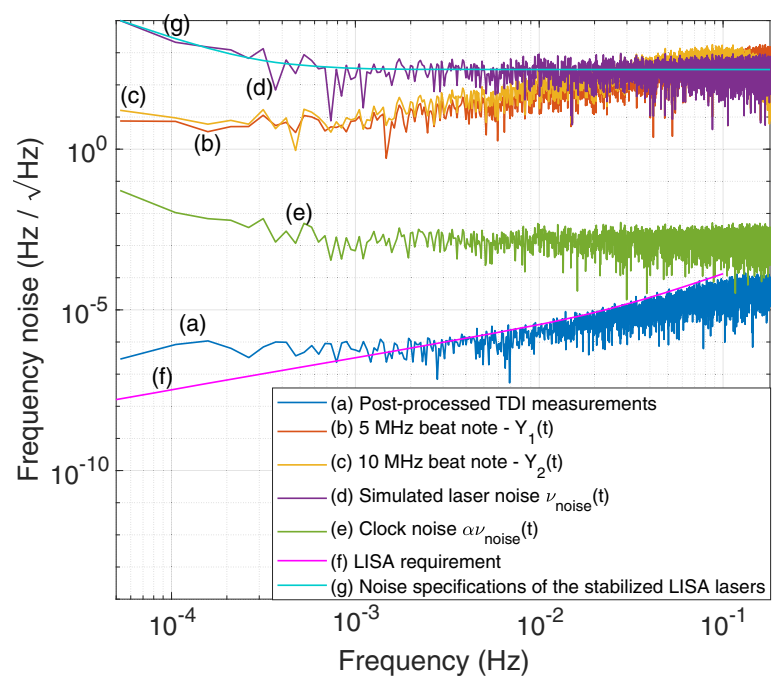

FIG. 8. OFC-based TDI experimental results. The LISA requirement is published in [6], and the noise specifications of the stabilized LISA laser are provided in [8].

levels have to be lowered by approximately 1.5 order of magnitude below $3 \times 10^{-3} \mathrm{~Hz}$. This would be possible by improving our test setup using an ultralow expansion bench in a vacuum chamber, much similar to that of the LISA optical test bench [8]. In addition, the long-term stability of the laser used also needs to be improved by 1.5 order of magnitude.

To understand the expected performance of the combbased second generation TDI scheme when applied to the actual LISA scenario, we performed a simulation of the best theoretically achievable performance. Figure 9 presents the simulation results for $\Delta \nu_{1}=5 \mathrm{MHz}, \Delta \nu_{2}=10 \mathrm{MHz}$, $T_{1}=2.7$ sec., $T_{2}=5.4$ sec., and $\alpha=\frac{f_{0}}{\nu_{0}}=5.17 \times 10^{-8}$ from a pure optical frequency divider comb.

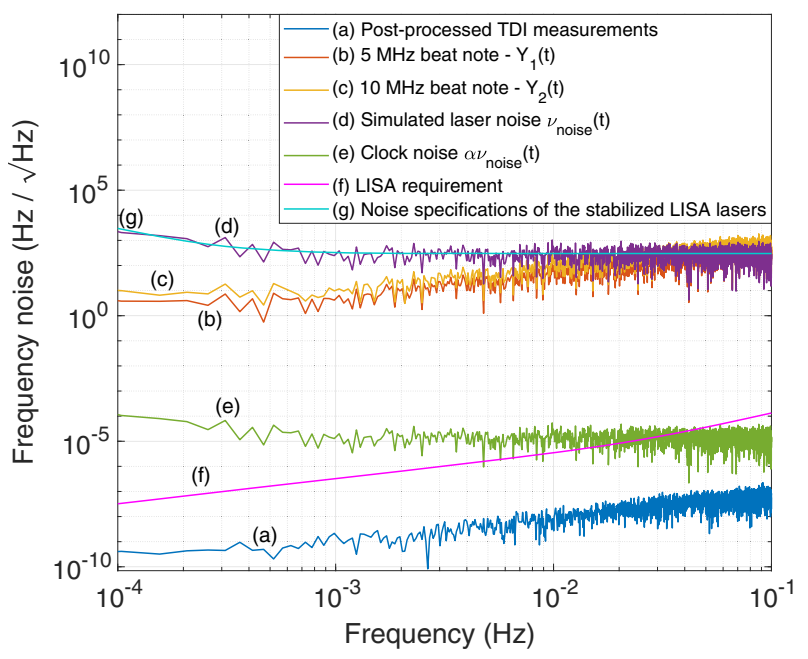

FIG. 9. Second generation TDI combination capability in the context of the LISA mission. 
As depicted in Fig. 9, the second generation TDI combination does not fully cancel the clock noise that is coherently transferred from the stabilized LISA laser. This is because of the approximation used for obtaining the TDI combination mentioned in Sec. II. However, the resulting errors are insignificant for all practical matter in the LISA applications. Indeed, as we can see in Fig. 9, a postprocessed TDI signal can meet the LISA noise requirement within the entire LISA bandwidth with over 2 orders of magnitude margins to spare. Based on the theoretical analysis and the experimental demonstrations reported here, we conclude that OFC technique applied to TDI can efficiently meet the LISA noise requirements with ample margins.

\section{CONCLUSIONS}

We have experimentally demonstrated the effectiveness of the OFC-based TDI for simultaneously canceling both laser and clock noises in unequal-arm space interferometry. This method relies on a self-referenced OFC locked to the ranging laser, with the OFC repetition rate rf signal used as a local oscillator for the heterodyne phase/frequency measurements. Using a realistic experimental implementation of the LISA unequal-arm interferometer, we were able to cancel the laser noise in the heterodyne measurements by 7 orders of magnitude down to the measurement noise floor of the laboratory setup. Moreover, we have also shown through a numerical simulation that this approach is capable of suppressing the laser and clock noises in the postprocessed TDI measurements below the LISA noise requirement with margins of over 2 orders of magnitude over the entire LISA bandwidth.

The implementation of this technique with LISA would lead to several significant simplifications of its payload while increasing the hardware robustness and reliability. With the deployment of an OFC, one eliminates the need of the clock frequency up-conversion to $1 \mathrm{GHz}$ and laser modulation to transfer the clock noise between the spacecraft. Accordingly, the heterodyne sideband-to-sideband measurements needed by the clock signal recovery and calibration algorithm are no longer required. Finally, the LISA baseline USO is no longer needed. Besides LISA, the comb-based second-generation TDI technique can be incorporated in the design of future space-borne gravitational wave detection interferometers [31,32].

\section{ACKNOWLEDGMENTS}

We acknowledge the financial support provided by the National Aeronautics and Space Administration (NASA) under the Astrophysics Research and Analysis Program (APRA)-Research Opportunities in Space and Earth Science (ROSES). M. T. also acknowledges support from F. A.P.E. S. P. (São Paulo, Brazil-Process No. 2019/ 07131-0) through a Visiting Professorship at the National Institute for Space Research (INPE), while this document was prepared. This research was performed at the Jet Propulsion Laboratory, California Institute of Technology, under contract with NASA. We would also like to thank Lin Yi for kindly providing us the ultrastable laser signal used in our experiment.
[1] A. Einstein and N. Rosen, On gravitational waves, J. Franklin Inst. 223, 43 (1937).

[2] K.S. Thorne, Gravitational Radiation (Cambridge University Press, New York, 1987), Vol. 330.

[3] The LIGO project website can be found at the following URL: http://www.ligo.caltech.edu/.

[4] B. P Abbott et al., Observation of Gravitational Waves from a Binary Black Hole Merger, Phys. Rev. Lett. 116, 061102 (2016).

[5] K. Danzmann et al., LISA Laser Interferometer Space Antenna-A proposal in response to the ESA call for L3 mission concepts, ESA publication, https://www.lisamission .org/?q=articles/lisa-mission/lisa-mission-proposal-13 (2017).

[6] LISA Science Study Team, LISA Science Requirements Document, ESA publication, https://www.cosmos.esa.int/ web/lisa/lisa-documents (2018).

[7] M. Tinto and J. W. Armstrong, Cancellation of laser noise in an unequal-arm interferometer detector of gravitational radiation, Phys. Rev. D 59, 102003 (1999).

[8] K. McKenzie, R. E. Spero, and D. A. Shaddock, Performance of arm locking in LISA, Phys. Rev. D 80, 102003 (2009).
[9] LISA mission description, https://www.cosmos.esa.int/web/ lisa/mission-description (2018).

[10] M. Tinto and S. V. Dhurandhar, Time-delay interferometry, Living Rev. Relativity 17, 6 (2014).

[11] R. W. Hellings, Elimination of clock jitter noise in spaceborne laser interferometers, Phys. Rev. D 64, 022002 (2001).

[12] M. Tinto, F. B. Estabrook, and J. W. Armstrong, Time-delay interferometry for LISA, Phys. Rev. D 65, 082003 (2002).

[13] G. de Vine, B. Ware, K. McKenzie, R. E. Spero, W. M. Klipstein, and D. A. Shaddock, Experimental Demonstration of Time-Delay Interferometry for the Laser Interferometer Space Antenna, Phys. Rev. Lett. 104, 211103 (2010).

[14] M. Tinto and O. Hartwig, Time-delay interferometry and clock-noise calibration, Phys. Rev. D 98, 042003 (2018).

[15] D. A. Shaddock, M. Tinto, F. B. Estabrook, and J. W. Armstrong, Data combinations accounting for LISA spacecraft motion, Phys. Rev. D 68, 061303(R) (2003).

[16] M. Tinto, F. B. Estabrook, and J. W. Armstrong, Time delay interferometry with moving spacecraft arrays, Phys. Rev. D 69, 082001 (2004). 
[17] S. J. Mitryk, G. Mueller, and J. Sanjuan, Hardware-based demonstration of time-delay interferometry and TDIranging with spacecraft motion effects, Phys. Rev. D 86, 122006 (2012).

[18] M. Tinto and N. Yu, Time-delay interferometry with optical frequency comb, Phys. Rev. D 92, 042002 (2015).

[19] T. Wilken, M. Lezius, T. W. Hänsch, A. Kohfeldt, A. Wicht, V. Schkolnik, M. Krutzik, H. Duncker, O. Hellmig, P. Windpassinger, K. Sengstock, A. Peters, and R. Holzwarth, A frequency comb and precision spectroscopy experiment in space, in CLEO: 2013, OSA Technical Digest (online) (Optical Society of America, 2013), paper AF2H.5.

[20] J. Lee, K. Lee Y. S. Jang, H. Jang, S. Han, S. H. Lee, K. I. Kang, C. W. Lim, Y. J. Kim, and S. W. Kim, Testing of a femtosecond pulse laser in outer space, Sci. Rep. 4, 5134 (2014).

[21] M. Lezius, T. Wilken, C. Deutsch, M. Giunta, O. Mandel, A. Thaller, V. Schkolnik, M. Schiemangk, A. Dinkelaker, A. Kohfeldt, A. Wicht, M. Krutzik, A. Peters, O. Hellmig, H. Duncker, K. Sengstock, P. Windpassinger, K. Lampmann, T. Hlsing, T. W. Hnsch, and R. Holzwarth, Space-borne frequency comb metrology, Optica 3, 1381 (2016).

[22] J. L. Hall, Nobel lecture: Defining and measuring optical frequencies, Rev. Mod. Phys. 78, 1279 (2006).

[23] S. A. Diddams, D. J. Jones, J. Ye, S. T. Cundiff, J. L. Hall, J. K. Ranka, R. S. Windeler, R. Holzwarth, T. Udem, and T. W. Hnsch, Direct Link Between Microwave and Optical Frequencies with a $300 \mathrm{THz}$ Femtosecond Laser Comb, Phys. Rev. Lett. 84, 5102 (2000).

[24] L. S. Ma, Z. Bi, A. Bartels, L. Robertsson, M. Zucco, R. S. Windeler, G. Wilpers, C. Oates, L. Hollberg, and S. A. Diddams, Optical frequency synthesis and comparison with uncertainty at the $10^{-19}$ level, Science 303, 1843 (2004).
[25] J. A. Barnes, A. R. Chi, L. S. Cutler, D. J. Healey, D. B. Leeson, T. E. Mcgunigal, J. A. Mullen, W. L. Smith, R. L. Sydnor, R. F. C. Vessot, and G. M. R. Winkler, Characterization of frequency stability, IEEE Trans. Instrum. Meas. IM-20, 105 (1971).

[26] P. D. Haye, A. Coillet, T. Fortier, K. Beha, D. C. Cole, K. Y. Yang, H. Lee, K. J. Vahala, S. B. Papp, and S. A. Diddams, Phase-coherent microwave-to-optical link with a self-referenced microcomb, Nat. Photonics 10, 516 (2016).

[27] V. Brasch, E. Lucas, J. D. Jost, M. Geiselmann, and T. J. Kippenberg, Self-referenced photonic chip soliton Kerr frequency comb, Light Sci. Appl. 6, e16202 (2017).

[28] M. Tinto, D. A. Shaddock, J. Sylvestre, and J. W. Armstrong, Implementation of time-delay interferometry for LISA, Phys. Rev. D 67, 122003 (2003).

[29] B. S. Sheard, M. B. Gray, D. E. McClelland, and D. A. Shaddock, Laser frequency stabilization by locking to a LISA arm, Phys. Lett. A 320, 9 (2003).

[30] E. Rubiola, E. Salik, S. Huang, N. Yu, and L. Maleki, Photonic-delay technique for phase-noise measurement of microwave oscillators, J. Opt. Soc. Am. 22, 987 (2005).

[31] W.-R. Hu and Y.-L. Wu, The Taiji program in space for gravitational wave physics and the nature of gravity, Natl. Sci. Rev. 4, 685 (2017).

[32] J. Luo, L.-S. Chen, H.-Z. Duan, Y.-G. Gong, S. Hu, J. Ji, Q. Liu, J. Mei, V. Milyukov, M. Sazhin, C.-G. Shao, V. T. Toth, H.-B. Tu, Y. Wang, Y. Wang, H.-C. Yeh, M.-S. Zhan, Y. Zhang, V. Zharov, and Z.-B. Zhou, rTianQin: A space-borne gravitational wave detector, Classical Quantum Gravity 33, 035010 (2016). 